\title{
Exchange-bias-induced double-shifted magnetization curves in Co biaxial films
}

\author{
Chih-Huang Lai and Yung-Hung Wang \\ Department of Materials Science \& Engineering, National Tsing Hua University, HsinChu, Taiwan \\ Ching-Ray Chang \\ Department of Physics, National Taiwan University, Taipei, Taiwan \\ Jyh-Shinn Yang \\ Institute of Optoelectronic Sciences, National Taiwan Ocean University, Keelung, Taiwan
}

Y. D. Yao

Institute of Physics, Academia Sinica, Taipei, Taiwan

(Received 5 April 2001; published 13 August 2001)

\begin{abstract}
Ferromagnetic films of fcc (001) Co, epitaxially grown on a NiMn antiferromagnetic underlayer, exhibit double-shifted magnetization curves. Exchange coupling not only provides a unidirectional bias field but also induces a uniaxial anisotropy, breaking the biaxial symmetry of Co. The double-shifted magnetization loops appear at certain range of Co thickness, in which both the shift field and exchange-bias field decreases as the Co film thickness increases. An interesting starlike astroid was found and it suggested that an interfacial biquadratic coupling results in double-shifted magnetization curves.
\end{abstract}

DOI: 10.1103/PhysRevB.64.094420

PACS number(s): 75.70.Cn, 75.30.Gw, 75.30.Et, 75.60.Jk

Exchange coupling between ferromagnetic (FM) and antiferromagnetic (AFM) materials has attracted great research interest during the past several years. ${ }^{1-6}$ The effective bias field on a FM thin film produced by the interfacial exchange with an AFM film is very useful in the information storage industry, e.g., magnetoresistive heads or magnetic random access memories. ${ }^{7}$ For the metal bias film, the epitaxial film is not easy to grow and the spin structure is also more complicated. Therefore, even though most of biasing films are metallic AFM's, the basic phenomena of metallic biasing films are still less understood than the insulating bias films. ${ }^{8}$

The physical origin of exchange bias was usually from the short-range exchange coupling at interface. Many experimental and theoretical results suggest that a strong dependence of the exchange-bias field on the interface spin configuration. The positive exchange bias in $\mathrm{FeF}_{2} / \mathrm{Fe}$ (Ref. 9) indicated a spin-flop interface state ${ }^{10,11}$ as well as existence of a domain wall formed at interface. ${ }^{12}$ For metal AFM films, Jungblut et al. ${ }^{13}$ also showed that in epitaxial $\mathrm{NiFe} / \mathrm{FeMn}$ bilayers, the magnetic moments of $\mathrm{NiFe}$ tended to orient perpendicular to the easy axis of FeMn. It has been also suggested that besides the unidirectional exchange anisotropy, effect of surface roughness and the relative orientation of FM and AFM spins at interface can induce biquadratic exchange anisotropy as observed in magnetic multilayers. ${ }^{14}$ Usually the exchange bias of the hysteresis loop was believed from the bilinear coupling (unidirectional anisotropy) between FM and uncompensated interfacial AFM spins, ${ }^{15}$ while the variation of the coercivity could be from the biquadratic coupling (uniaxial anisotropy) due to interfacial spin-flop state. ${ }^{11}$ Angular dependence of the exchange coupling in $\mathrm{NiFe} / \mathrm{CoO}$ bilayers revealed that the odd and even terms of the total anisotropy energy lead to exchange-bias field and coercivity, respectively. ${ }^{16}$ Dual-shifted magnetization curves have been observed in epitaxial Fe/MnPd (Ref. 17) and NiO/NiFe (Ref.
18) systems, and qualitative analyses by using StonerWohlfarth model provide a preliminary understanding for the magnetization reversal with complex anisotropies involved in the exchange-bias systems. However, detailed numerical fittings for angular dependence of experimental magnetization curves have not been reported to clearly illustrate the origin of the induced anisotropies, and to explain the asymmetrical loops, recently observed in exchange-bias system. $^{19,20}$

On the other hand, a hysteresis loop of fcc (001) Co, observed with the random steps at interface or miscut substrates, showed a uniaxial symmetry with locally breaking the fourfold symmetry. ${ }^{21,22}$ It was reported that the hysteresis loop along one of the biaxial axis would split into doubleshifted magnetization curves. Moreover, Weber et al. ${ }^{23}$ demonstrated that these shift fields could be enhanced with an in-plane transverse field. Magnetic anisotropy constants could be then determined from the double-shifted loop by measuring the shift field $H_{\mathrm{sh}}$ and the slope around $H=0 .^{24}$ Indeed, the double-shifted magnetization curves were predicted from numerical calculation of coherent rotation with some specific values of the quartic term of uniaxial anisotropy quite long ago. ${ }^{25}$ With the variation of quartic anisotropy, it has been theoretically shown that the magnetization curve along the hard axis is from anhysteresis to doubleshifted loops and then becomes square loops. However, the associated nucleation threshold is from the regular astroid to the astroid with extra cusps along the hard axis ${ }^{26,27}$ and finally evolves into the modified astroid with the starlike shape. $^{25}$

In this article, we make an epitaxial (001) NiMn/Co film to study the exchange-coupling-induced symmetry breaking on the biaxial fcc (001) Co film. We found that doubleshifted magnetization curves appeared, and could be tuned by several parameters, e.g., the variation of Co film thickness 


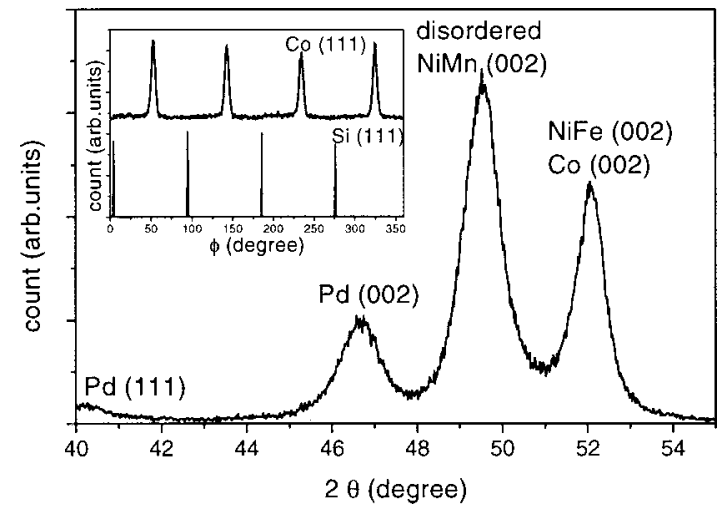

FIG. 1. X-ray symmetric $\theta-2 \theta$ and asymmetric $\phi$ scan for the as-deposit $\mathrm{Si}(100) / \mathrm{Cu}(15 \mathrm{~nm}) / \mathrm{Ni}_{80} \mathrm{Fe}_{20}(35 \mathrm{~nm}) / \mathrm{NiMn}(50 \mathrm{~nm}) /$ $\mathrm{Co}(15 \mathrm{~nm}) / \mathrm{Pd}(15 \mathrm{~nm})$.

and the field-annealed time. The double-shifted magnetization curves only appear in the certain range of Co thickness, in which the shift field decreases as the thickness increases and is proportional to the exchange-bias field. Outside this specific range of Co thickness, the shifted magnetization curves disappear but the exchange bias still remains. Wellfitted numerical calculations of the experimental data show that a biquadratic coupling presented within a NiMn/Co system. From the analysis of the angular dependence of hysteresis loop and modified astroid with starlike shape, ${ }^{25}$ we conclude that induced anisotropies are interfacial. We notice that double-shifted magnetization curves exist only with a narrow range of the effective biquadratic coupling strength. In particular, asymmetrical loops are also observed with the applied field deviated from $\langle 110\rangle$ of the Co film.

Samples with the structure $\operatorname{Si}(100) / \mathrm{Cu}(15 \mathrm{~nm}) /$ $\mathrm{Ni}_{80} \mathrm{Fe}_{20}(35 \mathrm{~nm}) / \mathrm{NiMn}(50 \mathrm{~nm}) / \mathrm{Co}(t \mathrm{~nm}) / \mathrm{Pd}(15 \mathrm{~nm}) \quad$ were grown by an $e$-beam evaporation system, and the thickness of Co was varied from $5 \mathrm{~nm}$ to $25 \mathrm{~nm}$. Cu was used to initiate (001) epitaxial growth and thick $\mathrm{Ni}_{80} \mathrm{Fe}_{20}$ was used to prevent $\mathrm{Cu}$ from diffusing into $\mathrm{NiMn} .{ }^{28} \mathrm{Pd}$ was used for the capping layer. NiMn was grown at $120^{\circ} \mathrm{C}$, and others were grown at room temperature. No external field was applied during the depositions, but after depositions, samples were field-annealed ex situ in the field of $1000 \mathrm{Oe}$ at $280^{\circ} \mathrm{C}$ in the vacuum to induce the exchange-bias field. The details of sample preparation were reported elsewhere. ${ }^{28}$ The structure was characterized by x-ray diffraction, and the hysteresis loops measured by both VSM and magneto-optical Kerr effect (MOKE) at room temperature. Due to the short penetration depth of laser $(\sim 30 \mathrm{~nm})$, MOKE showed only Co loops and thus the contribution of $\mathrm{Ni}_{80} \mathrm{Fe}_{20}$ can be differentiated from Co loops in VSM data.

The x-ray $\theta-2 \theta$ scan for the as-deposit sample, shown in Fig. 1, shows (002) peaks of disordered $\mathrm{NiMn}, \mathrm{NiFe}$, and $\mathrm{Co}$, indicating a good out-of-plane (001) texture. Furthermore, the asymmetric x-ray $\phi$ scan of Co (111) verifies that Co was grown on NiMn epitaxially, and the epitaxial relationships are $\mathrm{Si}(001)\|\mathrm{NiFe}(001)\| \mathrm{NiMn}(001) \| \mathrm{Co}(001)$ and $\mathrm{Si}[110]|\mathrm{NiFe}[010]| \mathrm{NiMn}[010] \| \mathrm{Co}[010]$. Both VSM and MOKE show no exchange-bias field acting on $\mathrm{Ni}_{80} \mathrm{Fe}_{20}$ and $\mathrm{Co}$, which implies that as-deposit NiMn is not an antiferro-

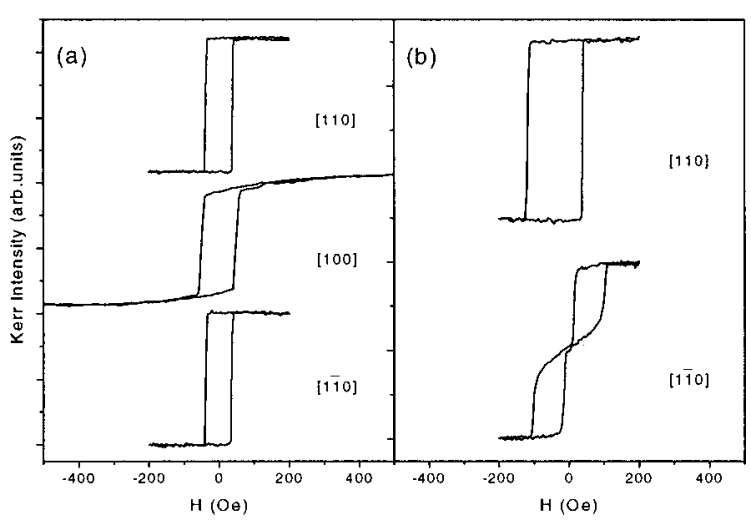

FIG. 2. Hysteresis loops of fcc (001) NiMn/Co(15 nm) measured at $300 \mathrm{~K}$. (a) Before field annealing, the biaxial anisotropy appeared along two orthogonal directions. (b) After 1000 Oe fieldannealing along the [110], the biaxial anisotropy disappeared, and the shifted loop appeared along the [110]. Note the double-shifted magnetization curves appeared in the $[1 \overline{1} 0]$.

magnetic phase. The hysteresis loops of as-deposit $\operatorname{Co}(15$ $\mathrm{nm}$ ) on NiMn measured by MOKE show a perfect biaxial anisotropy. We did not observe any influences on Co anisotropy from either NiM nor interface [Fig.2(a)]. The saturation field of hard axis (Co [100]) is about 400 Oe, and the crystalline anisotropy constant can be estimated by considering the formula $K_{1}=\left(H_{s} \times M_{s}\right) / 2$, where the $H_{s}$ is the saturation field and $M_{s}$ is the saturation magnetization of Co film. For $M_{s}=1400 \mathrm{emu} / \mathrm{cm}^{3}$, the crystalline anisotropy constant $K_{1}$ is about $2.8 \times 10^{5} \mathrm{erg} / \mathrm{cm}^{3}$ for biaxial Co film which is about the same as reported data. ${ }^{29}$

After postannealing with an external field of $1000 \mathrm{Oe}$ along Co [110] at $280^{\circ} \mathrm{C}$, parts of NiMn transformed to an antiferromagetic phase. The details of phase transformation were reported elsewhere. ${ }^{30}$ The epitaxial relationship between layers was maintained after annealing. Figure 2(b) shows the hysteresis loops of 2-h annealed $\mathrm{NiMn} / \mathrm{Co}(15 \mathrm{~nm})$. The loop measured along Co [110] (the direction of external field during annealing) shows a square loop and exhibits an exchange-bias field of $41 \mathrm{Oe}$, but the loop measured along Co [1 $1 \overline{1} 0]$ shows double-shifted magnetization curves. With increasing the annealing time, the exchange-bias field increases, and saturates at $50 \mathrm{Oe}$ after $4 \mathrm{~h}$. However, the separation of the double-shifted loop $\left(2 H_{\mathrm{sh}}\right)$ still increases. Meanwhile, the coercivity of Co film increases with the increasing of the annealing time. Table I shows the exchangebias field, coercivity, and shift field of $\mathrm{NiMn} / \mathrm{Co}(15 \mathrm{~nm})$ for different annealing time.

TABLE I. Effect of field-annealing time on coercivity $\left(H_{c}\right)$, exchange bias field $\left(H_{e}\right)$, and shift field $\left(H_{\mathrm{sh}}\right)$.

\begin{tabular}{lcccccc}
\hline \hline & $0.5 \mathrm{~h}$ & $1 \mathrm{~h}$ & $2 \mathrm{~h}$ & $3 \mathrm{~h}$ & $4 \mathrm{~h}$ & $8 \mathrm{~h}$ \\
\hline$H_{e}(\mathrm{Oe})$ & 38 & 38 & 41 & 46 & 50 & 50 \\
$H_{c}(\mathrm{Oe})$ & 52 & 70 & 80 & 94 & 98 & 102 \\
$H_{\mathrm{sh}}(\mathrm{Oe})$ & 50 & 52 & 57 & 60 & 62 & 68 \\
\hline \hline
\end{tabular}


TABLE II. Effect of field-annealing time on $K_{1}$ and $K_{b} . K_{1}$ and $K_{b}$ are obtained from the numerical fitting of hysteresis loops.

\begin{tabular}{ccccccc}
\hline \hline & $0.5 \mathrm{~h}$ & $1 \mathrm{~h}$ & $2 \mathrm{~h}$ & $3 \mathrm{~h}$ & $4 \mathrm{~h}$ & $8 \mathrm{~h}$ \\
\hline $\begin{array}{c}K_{1} \\
\left.\mathrm{erg} / \mathrm{cm}^{3}\right)\end{array}$ & 1.276 & 1.408 & 1.401 & 1.529 & 1.559 & 1.571 \\
$\begin{array}{c}K_{b} / t \\
\left(10^{4} \mathrm{erg} / \mathrm{cm}^{3}\right)\end{array}$ & 3.003 & 3.323 & 3.837 & 3.528 & 3.319 & 4.115 \\
\hline \hline
\end{tabular}

For fcc (001) Co film, the biaxial crystalline anisotropy energy is $K_{1} \cos ^{2} \theta \sin ^{2} \theta$, where $K_{1}$ is the cubic anisotropy constant and $\theta$ is the angle between magnetization and easy axis. Double-shifted magnetization curves were observed with the random steps at interface or miscut substrates because of the fourfold symmetry locally broken by a stepinduced uniaxial symmetry, $K_{s} \cdot{ }^{23}$ The formation of the double-shifted loops can be understood with the StonerWohlfarth theory of the appropriate ratio of the $K_{1}+K_{s}$ to $K_{1} \cdot{ }^{25}$ It has also showed that the shift field of double loops was enhanced with an in-plane external bias field along the uniaxial direction of Co films. ${ }^{23,24}$ In our study, since the as-deposit $\mathrm{NiMn} / \mathrm{Co}$ system manifested only the biaxial properties of fcc Co film, the double-shifted magnetization curves of field-annealed fcc (001) NiMn/Co system can then be understood as the break of fourfold symmetry in Co films by the exchange coupling to NiMn. Exchange coupling between NiMn and Co induces an internal bias field as well as a biquadratic coupling. This biquadratic exchange coupling arising from the interface produced a uniaxial anisotropy along the applied field direction during annealing. This induced anisotropy enhanced the coercivity along the easy axis, and resulted in the double-shifted loops along the hard axis.

The loops of Co film can be well fitted with the coherent rotation model with the consideration of three energies: (1) the Zeeman energy, $-\mathbf{M}_{s} \cdot \mathbf{H} t$, (2) the magnetocrystalline anisotropy of Co, $K_{1} t \cos ^{2} \theta \sin ^{2} \theta$, and (3) the interfacial exchange anisotropy energy, $-K_{e} \cos \theta-K_{b} \cos ^{2} \theta$ from NiMn. The energy per unit area, $E\left(\mathrm{erg} / \mathrm{cm}^{2}\right)$, could be expressed as

$$
E=-\mathbf{M}_{s} \cdot \mathbf{H} t+K_{1} t \cos ^{2} \theta \sin ^{2} \theta-K_{e} \cos \theta-K_{b} \cos ^{2} \theta,
$$

where $t$ is the thickness of $\mathrm{Co} ; \mathbf{H}$ is the applied field; $K_{1}$ is the crystalline anisotropy constant of fcc Co; $K_{e}$ is the exchange unidirectional constant and is equal to $M_{s} H_{e} t . H_{e}$ is the exchange bias field in $\mathrm{NiMn} / \mathrm{Co} ; K_{b}$ is the biquadratic coupling anisotropy constant. $\theta$ is the angle between the magnetization and the field-annealed direction. All the magnetic anisotropy constants could be determined from the numerical calculation of Stoner-Wohlfarth model. ${ }^{25}$ Table II shows those anisotropy constants of $\mathrm{NiMn} / \mathrm{Co}(15 \mathrm{~nm})$.

As-deposit sample exhibits a perfect biaxial anisotropy, but field-annealed sample brings into an extra uniaxial anisotropy (i.e., biquadratic coupling $-K_{b} \cos ^{2} \theta$ ). When annealing time smaller than $2 \mathrm{~h}, K_{b}$ increased with increasing annealing time, but when annealing time exceeds $2 \mathrm{~h}, K_{b}$ changes irregularly. During the first $2 \mathrm{~h}$, the amount of antiferromagnetic phase increases, which may enhance the spin- flop state at the interface, leading to an increase in biquadratic coupling. But when annealing time was greater than 2 $\mathrm{h}$, substantial interdiffusion occurred; ${ }^{30}$ therefore, it may change the crystalline anisotropy of Co film, and cause the irregular change of $K_{b}$.

We have repeated the previous experimental procedure for the samples with the different thickness of Co film. We found that the double-shifted magnetization curves only exists at Co film thickness between $10 \mathrm{~nm}$ and $15 \mathrm{~nm}$. Indeed, the previous numerical calculations ${ }^{25}$ already showed that only a narrow range of the $\left(K_{1}+K_{b}\right) / K_{1}$ will induce the doubleshifted magnetization curves; otherwise, the hard axis loops are anhysteresis. In our $\mathrm{NiMn} / \mathrm{Co}$ system, since $K_{b}$ varies with the Co film thickness, it is quite reasonable that the double-shifted magnetization curves only appear at a very specific range of Co film thickness. The exchange bias field and $H_{\mathrm{sh}}$ decrease with the increasing Co thickness, and it suggests that their origins are interfacial in NiMn/Co system.

The experimental data reveal the detailed features of the coherent rotation. Both the reversible and irreversible movements of the magnetization are almost identical to the numerical calculations. Figure 3 shows that the hysteresis loops
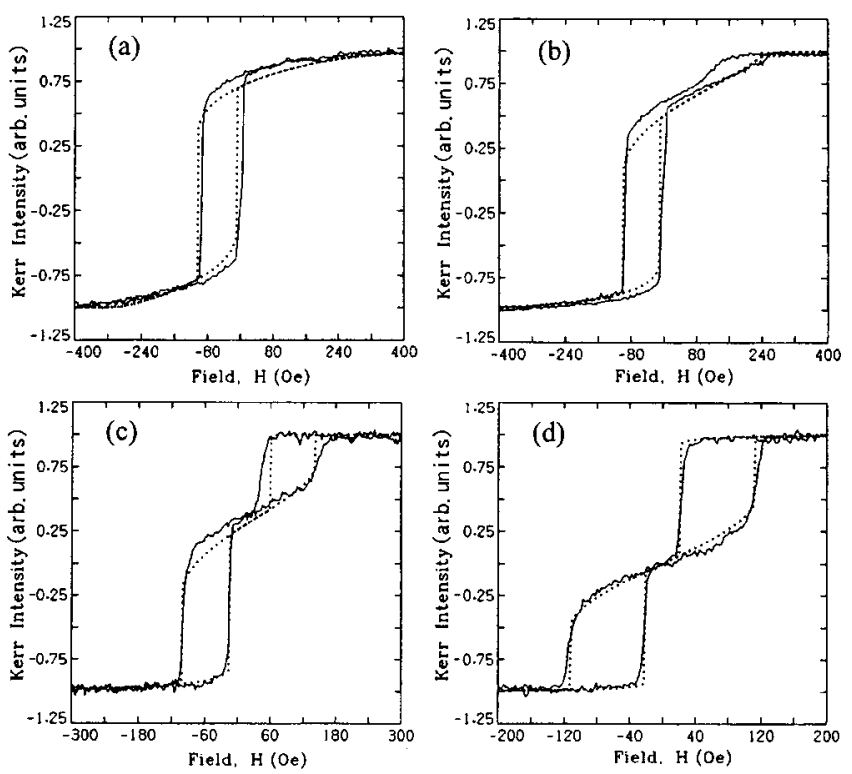

FIG. 3. Hysteresis loops of $\mathrm{NiMn} / \mathrm{Co}(15 \mathrm{~nm})$ with four different deviations from the [110] axis are shown, i.e., (a) $45^{\circ}$, (b) $60^{\circ}$, (c) $75^{\circ}$, and (d) $90^{\circ}$. Solid lines represent the experimental data, which are taken from the MOKE measurement, and dotted lines are numerical calculations of coherent rotation. The material parameters used in our calculations are $K_{1}=1.57 \times 10^{5} \mathrm{erg} / \mathrm{cm}^{3}, K_{b} / t=4.12$ $\times 10^{4} \mathrm{erg} / \mathrm{cm}^{3}$, and $M_{s}=1400 \mathrm{emu} / \mathrm{cm}^{3}$. 
of 8-h annealed NiMn/Co $(15 \mathrm{~nm})$ at applied field with the different deviations from the [110] axis of Co. Beside the double-shifted loops along [110] axis, we also observed asymmetrically shaped hysteresis loops. We can reproduce both the double shifted loops and asymmetrically loops from the coherent rotation model. This is possibly because that $\mathrm{NiMn} / \mathrm{Co}$ system is epitaxial and the magnetization rotates in unison within the Co films. Figure 3 shows the calculated loops with $K_{1}=1.57 \times 10^{5} \mathrm{erg} / \mathrm{cm}^{3}$ and $K_{b} / t=4.12$ $\times 10^{4} \mathrm{erg} / \mathrm{cm}^{3}$. The fitting goes reasonably well with the experimental measurements. Similar asymmetrical loops with exchange bias have been reported recently, but the physical origins of their asymmetrical behavior are quite different. ${ }^{19,20}$ We also studied the criteria of nucleation fields. The measured switching fields at different deviation angle were reduced into two orthogonal components, $H_{x}$ and $H_{y}$. The modified astroid with a starlike shape ${ }^{25}$ was obtained experimentally (Fig. 4), even though some extra cusps on the easy axis with quartic anisotropy of small particles were identified before. ${ }^{26,27}$ Comparing with the unshifted astroid, ${ }^{25}$ the nucleation thresholds in our modified astroid is asymmetric and some cusps become invisible due to the eccentricity of applied field and the modified astroid. The asymmetric magnetization curves may result from the same origin. The experimental data are compressed at small angles in comparison with the best-fitted modified astroid (Fig. 4). Similar deformation of the nucleation threshold was found along the easy axis for the possibility of incoherent reversal of magnetization. ${ }^{26,31}$

In summary, we have studied the hysteresis behavior of the epitaxial (001) NiMn/Co system. Numerical fitting shows magnetization reversal of $\mathrm{NiMn} / \mathrm{Co}$ is nucleation, instead of domain wall movement observed at the wedged-NiFe/FeMn bilayer. ${ }^{32}$ The double-shifted magnetization curves were observed along the axis perpendicular to the field-annealing direction, which can be attributed to the biquradatic coupling. The biquradatic anisotropy originated from interfacial coupling, plausibly induced from the interfacial spin-flop state, breaks locally the biaxial anisotropy of fcc (001) Co. In an epitaxial NiMn/Co system, we found that the magnetization curves along different directions can be well fitted by

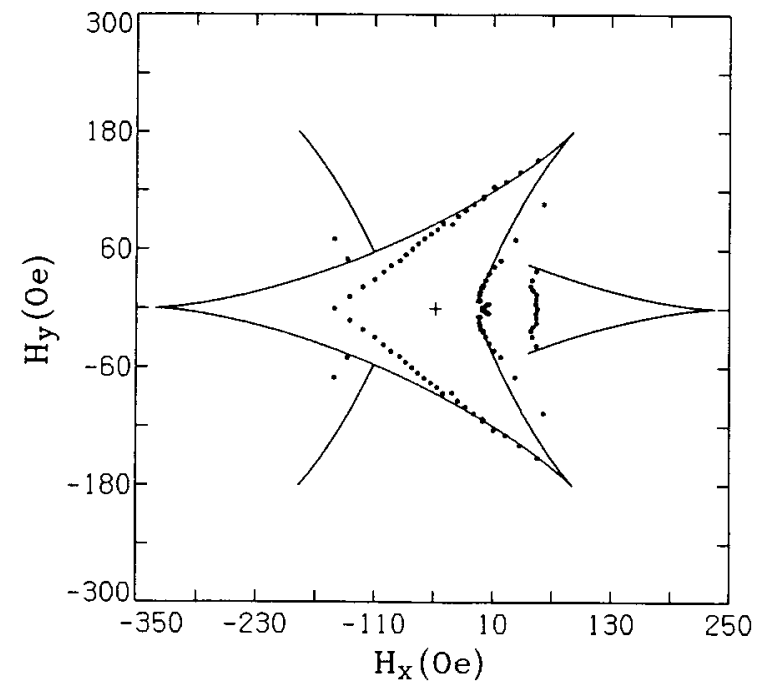

FIG. 4. Modified astroid of the instability conditions, where $H_{x}$ and $H_{y}$ are the components of applied field parallel and perpendicular to the easy axis. For clarity, only the decreasing-field branch is shown here. The full circles are the experimental data of the nucleation fields, while the solid line is the locus of the instability conditions of Eq. (1). It should be noted that the astroid shifted under the exchange-bias field and is asymmetric. Compression along the easy axis of experimental data indicated that the incoherent reversal mode appears in the $\mathrm{NiMn} / \mathrm{Co}$ system. The parameters used here are $K_{1}=1.57 \times 10^{5} \mathrm{erg} / \mathrm{cm}^{3}, \quad K_{b} / t=4.12 \times 10^{4} \mathrm{erg} / \mathrm{cm}^{3}, \quad$ and $M_{s}$ $=1400 \mathrm{emu} / \mathrm{cm}^{3}$.

numerical calculations of Stoner-Wohlfarth model, and the effective anisotropy constants can be tuned from the variation of Co thickness or annealing time. Moreover, with a biased modified astroid, the observed asymmetric loops can be understood and the double-shifted loops become controllable in the $\mathrm{Co} / \mathrm{NiMn}$ system by adjusting the ratio of $\left(K_{1}\right.$ $+K_{b}$ ) to $K_{1}$.

We would like to thank Dr. C. K. Lo for stimulated discussions and assistance on sample preparations. This work was supported by National Science Council of R.O.C. under Grants NSC.89-2112-M-007-067 and NSC.89-2112-M-019004.
${ }^{1}$ W. H. Meiklejohn and C. P. Bean, Phys. Rev. 102, 1413 (1956); 105, 904 (1957).

${ }^{2}$ A. P. Malozemoff, Phys. Rev. B 35, 3679 (1987).

${ }^{3}$ J. Nogués and I. K. Schuller, J. Magn. Magn. Mater. 192, 203 (1999).

${ }^{4}$ C. Leighton, J. Nogués, B. J. Jönsson-Åkerman, and I. K. Schuller, Phys. Rev. Lett. 84, 3466 (2000).

${ }^{5}$ H. Xi, M. H. Kryder, and R. M. White, Appl. Phys. Lett. 74, 2687 (1999).

${ }^{6}$ Y. Ijiri, J. A. Borchers, R. W. Erwin, S.-H. Lee, P. J. van der Zaag, and R. M. Wolf, Phys. Rev. Lett. 80, 608 (1998).

${ }^{7}$ D. D. Tang, P. K. Wang, V. S. Speriosu, S. Le, and K. K. Kung, IEEE Trans. Magn. 33, 3283 (1997).

${ }^{8}$ A. E. Berkowitz and K. Takano, J. Magn. Magn. Mater. 200, 552
(1999).

${ }^{9}$ J. Nogués, D. Lederman, T. J. Moran, and I. K. Schuller, Phys. Rev. Lett. 76, 4624 (1994).

${ }^{10}$ N. C. Koon, Phys. Rev. Lett. 78, 4865 (1997).

${ }^{11}$ Kentaro Takano, R. H. Kodama, A. E. Berkowitz, W. Cao, and G. Thomas, Phys. Rev. Lett. 79, 1130 (1997).

${ }^{12}$ C. Mauri, H. C. Siegmann, P. S. Bagus, and E. Key, J. Appl. Phys. 62, 3047 (1987).

${ }^{13}$ R. Jungblut, R. Coehoorn, M. Johnson, J. ann de Stegge, and A. Reinders, J. Appl. Phys. 75, 6659 (1994).

${ }^{14}$ J. C. Slonczewski, Phys. Rev. Lett. 67, 3172 (1991).

${ }^{15}$ T. C. Schulthess and W. H. Butler, Phys. Rev. Lett. 81, 4516 (1998).

${ }^{16}$ T. Ambrose, R. L. Sommer, and C. L. Chien, Phys. Rev. B 56, 83 
(1997).

${ }^{17}$ Y. J. Tang, X. Zhou, X. Chen, B. Q. Liang, and W. S. Zhan, J. Appl. Phys. 88, 2054 (2000).

${ }^{18}$ R. P. Michel, A. Chaiken, C. T. Wang, and L. E. Johnson, Phys. Rev. B 58, 8566 (1998).

${ }^{19}$ V. I. Nikitenko, V. S. Gornakov, A. J. Shapiro, R. D. Shull, K. Liu, S. M. Zhou, and C. L. Chien, Phys. Rev. Lett. 84, 765 (2000).

${ }^{20}$ M. R. Fitzsimmons, P. C. Yashar, C. Leighton, J. Nogués, J. Dura, C. F. Majkrzak, and I. K. Schuller, Phys. Rev. Lett. 84, 3986 (2000).

${ }^{21}$ A. Berger, U. Linke, and H. P. Oepen, Phys. Rev. Lett. 68, 839 (1992).

${ }^{22}$ W. Weber, C. H. Back, A. Bischof, D. Pescia, and R. Allenspach, Nature (London) 374, 788 (1995).

${ }^{23}$ W. Weber, C. H. Back, A. Bischof, Ch. Wursch, and R. Allenspach, Phys. Rev. Lett. 76, 1940 (1996).

${ }^{24}$ W. Weber, R. Allenspach, and A. Bischof, Appl. Phys. Lett. 70, 520 (1997).
${ }^{25}$ Ching-Ray Chang, J. Appl. Phys. 69, 2431 (1991).

${ }^{26}$ W. Wernsdorfer, E. Bonet Orozco, K. Hasselbach, A. Benoit, B. Barbara, N. Demoncy, A. Loiseau, H. Pascard, and D. Mailly, Phys. Rev. Lett. 78, 1791 (1997).

${ }^{27}$ W. Wernsdorfer, E. Bonet Orozco, K. Hasselbach, A. Benoit, D. Mailly, O. Kubo, H. Nakano, and B. Barbara, Phys. Rev. Lett. 79, 4014 (1997).

${ }^{28}$ Y. H. Wang, C. H. Lai, C. K. Lo, and Y. D. Yao, J. Magn. Magn. Mater. 209, 119 (2000).

${ }^{29}$ P. Krams, B. Hillebrands, G. Guntherodt, and H. P. Oepen, Phys. Rev. B 49, 3633 (1994).

${ }^{30}$ C. H. Lai, Y. H. Wang, W. C. Lien, and C. K. Lo, IEEE Trans. Magn. 36, 2641 (2000).

${ }^{31}$ A. Aharoni, J. Appl. Phys. 30, 70S (1959); in Magnetism III, edited by G. T. Rado and H. Shul (Academic, New York, 1963), p. 407.

${ }^{32}$ S. M. Zhou, K. Liu, and C. L. Chien, Phys. Rev. B 58, R14717 (1998). 